\title{
Finite-time stability of switched systems with delayed arguments and nonlinear perturbations
}

\author{
Youliang $\mathrm{Fu}^{1}{ }^{\circledR}$, Naxin $\mathrm{Cui}^{1}{ }^{\circledR}$, Chenghui Zhang ${ }^{1}\left(\mathbb{C}\right.$, Tongxing $\mathrm{Li}^{* 2,3}{ }^{\circledR}$ \\ ${ }^{1}$ School of Control Science and Engineering, Shandong University, Jinan, Shandong 250061, P. R. China \\ ${ }^{2}$ LinDa Institute of Shandong Provincial Key Laboratory of Network Based Intelligent Computing, Linyi \\ University, Linyi, Shandong 276005, P. R. China \\ ${ }^{3}$ School of Information Science and Engineering, Linyi University, Linyi, Shandong 276005, P. R. China
}

\begin{abstract}
This paper is concerned with the problem of finite-time stability (FTS) of a class of switched systems with delayed arguments and nonlinear perturbations which are related not only with the current state and the delayed state but also with time $t$. Novel LyapunovKrasovskii functions are introduced, and a new finite-time stability criterion is derived by employing the average dwell time (ADT) approach and linear matrix inequality technique. An example is given to illustrate the effectiveness of the proposed method.
\end{abstract}

Mathematics Subject Classification (2010). 34D20, 37N35

Keywords. finite-time stability, Lyapunov-Krasovskii function, nonlinear perturbation, average dwell time

\section{Introduction}

Switched systems which belong to a special class of hybrid systems have received a great deal of attention, such as stability [5,8,9,14], controllability and observability [6]. This is due to the fact that switched systems have a number of applications in traffic control, mechanical control, and so forth. In particular, time-delay systems were investigated in $[12,18,19]$.

Most of the existing papers related to stability of switched system focus on Lyapunov asymptotic stability (LAS), which is defined over an infinite time interval. However, in practice, one is interested in not only system stability (usually in the sense of Lyapunov) but also a bound of system trajectories over a fixed short time interval, such as networked control systems [15]. In addition, a system could be Lyapunov stable but still completely useless because it possesses undesirable transient performances, such as the system with saturation elements in the control loop. To study the transient performances of a system, the concept of short time stability, i.e., FTS, was introduced in [1,4], and the proposed approach in [1] was very effective. In particular, a system is said to be FTS if, given a bound on the initial condition, its state remains within a prescribed bound in a fixed time interval. Note that FTS and LAS are independent concepts: a system could be

\footnotetext{
*Corresponding Author.

Email addresses: gllfyl@163.com (Y. Fu), cuinx@sdu.edu.cn (N. Cui), zchui@sdu.edu.cn (C. Zhang), litongx2007@163.com (T. Li)

Received: 16.03.2017; Accepted: 10.10.2018
} 
FTS but not LAS, and vice versa [2]. Finite time stability of switched delay systems were investigated in $[10,11,13,16,21]$. To mention a few, Lin et al. [10] considered the switched linear delay system with norm-bounded disturbance and obtained several stability criteria by employing multiple Lyapunov functions method. Recently, using the generalized Grönwall-Bellman inequality, Tian et al. [16] investigated finite-time stability of impulsive switched delay systems with nonlinear disturbances, where the disturbances are related not only with current state and the delayed state but also with time $t$.

Motivated by the works $[10,16]$, the FTS of switched delay systems with nonlinear perturbations is considered in this paper. The main contributions of this paper can be summarized as follows: $(i)$ a time-varying delay and nonlinear perturbations are added compared to systems studied in [10]; (ii) several more effective stability criteria are obtained in terms of linear matrix inequalities which differ from the method exploited in [16].

Notation 1.1. Throughout the paper, $\mathbb{R}^{n}$ denotes the $n$-dimensional Euclidean space with vector norm $\|\cdot\|, \mathbb{R}^{n \times m}$ is the set of all $n \times m$-dimensional real matrices. I denotes the identity matrix of appropriate dimensions, and the superscript " $T$ " stands for matrix transposition. The notation $P>0(\geq 0)$ means that $P$ is symmetric and positive (semipositive) definite. $\lambda_{\min }(A)$ and $\lambda_{\max }(A)$ denote the minimum and maximum eigenvalues of $A$, respectively.

\section{Problem description and preliminaries}

Consider the following switched delay system with nonlinear perturbations

$$
\left\{\begin{array}{l}
\dot{x}(t)=A_{\sigma(t)} x(t)+B_{\sigma(t)} x(t-h(t))+f_{\sigma(t)}(t, x(t), x(t-h(t))), \quad t \geq 0, \\
x(t)=\phi(t), \quad t \in\left[-h_{2}, 0\right],
\end{array}\right.
$$

where $x(t) \in \mathbb{R}^{n}$ is the state vector, $A_{\sigma(t)}$ and $B_{\sigma(t)}$ are constant real matrices, $\phi(t) \in$ $C^{1}\left(\left[-h_{2}, 0\right], \mathbb{R}^{n}\right)$ with $\|\phi\|=\sup _{t \in\left[-h_{2}, 0\right]}\{\|\phi(t)\|,\|\dot{\phi}(t)\|\}$, where $C\left(\left[-h_{2}, 0\right], \mathbb{R}^{n}\right)$ is the Banach space of continuous functions. $\sigma(t):[0,+\infty) \rightarrow \Lambda=\{1,2, \ldots, m\}$ is the switching signal which is a piecewise constant function depending on time $t$ or state $x(t)$, and $m$ is the number of subsystems. The delay $h(t)$ is time-varying and satisfies

$$
0 \leq h_{1} \leq h(t) \leq h_{2}, \quad \dot{h}(t) \leq \mu<1,
$$

where $\mu$ is a positive constant, $h_{1}$ and $h_{2}$ are constants representing the lower and upper bounds of the delay, respectively. $f_{i}(t, x(t), x(t-h(t))), i \in \Lambda$ are nonlinear perturbations satisfying

$$
\left\|f_{i}(t, x(t), x(t-h(t)))\right\| \leq a\|x(t)\|+b\|x(t-h(t))\|+\beta(t),
$$

where $\beta(t)$ satisfies $\int_{0}^{T} \beta^{2}(s) e^{-\alpha s} d s<\infty, \alpha, T, a$, and $b$ are positive constants.

Assumption 2.1. The state of switched delay system does not jump at switching instants, i.e., the trajectory $x(t)$ is continuous everywhere. Switching signal $\sigma(t)$ has finite switching number in any finite interval time.

Definition 2.2 (see [1]). Given three positive constants $c_{1}, c_{2}, T$ with $c_{1}<c_{2}$, a positive definite matrix $R$, and a switching signal $\sigma(t)$. If

$$
\sup _{\theta \in\left[-h_{2}, 0\right]} \varphi^{T}(\theta) R \varphi(\theta) \leq c_{1} \Rightarrow x^{T}(t) R x(t) \leq c_{2}, \quad t \in[0, T],
$$

then switched system (2.1) is said to be FTS with respect to $\left(c_{1}, c_{2}, T, \sigma(t), R\right)$. If $(2.4)$ holds under an arbitrary switching signal $\sigma(t)$, then switched system $(2.1)$ is said to be uniformly FTS with respect to $\left(c_{1}, c_{2}, T, R\right)$. 
Definition 2.3 (see [10]). For a switching signal $\sigma(t)$ and any $t_{2}>t_{1} \geq 0$, let $N_{\sigma}\left(t_{1}, t_{2}\right)$ denote the number of discontinuities of $\sigma(t)$ in the open interval $\left(t_{1}, t_{2}\right)$. We say that $\sigma(t)$ has an average dwell time (ADT) $\tau_{a}>0$ if $\tau_{a}$ satisfies

$$
N\left(t_{1}, t_{2}\right) \leq N_{0}+\frac{t_{2}-t_{1}}{\tau_{a}} \text {. }
$$

Without loss of generality, we choose $N_{0}=0$ in this paper.

\section{Main results}

In this section, we focus on FTS of switched system (2.1). First, consider system (2.1) without switching

$$
\left\{\begin{array}{l}
\dot{x}(t)=A x(t)+B x(t-h(t))+f(t, x(t), x(t-h(t))), \quad t \geq 0, \\
x(t)=\phi(t), \quad t \in\left[-h_{2}, 0\right] .
\end{array}\right.
$$

Choose a Lyapunov-Krasovskii function as follows:

$$
V(t)=V_{1}(t)+V_{2}(t)
$$

where

$$
V_{1}(t)=x^{T}(t) \widetilde{P}_{1} x(t), \quad V_{2}(t)=\int_{t-h(t)}^{t} x^{T}(s) e^{\alpha(t-s)} \widetilde{Q}_{1} x(s) d s,
$$

$\alpha$ is a nonnegative constant, $\widetilde{P}_{1}$ and $\widetilde{Q}_{1}$ are positive definite matrices to be determined.

Lemma 3.1 (see [20]). For any $x, y \in R^{n}$ and any positive definite matrix $P \in \mathbb{R}^{n \times n}$,

$$
2 y^{T} x \leq x^{T} P^{-1} x+y^{T} P y .
$$

Lemma 3.2. Suppose that there exist matrices $\widetilde{P}_{1}>0, \widetilde{Q}_{1}>0$, and a constant $\alpha \geq 0$ such that

$$
\Sigma_{1}=\left[\begin{array}{ccc}
\Sigma_{11} & \Sigma_{12} & \Sigma_{13} \\
* & \Sigma_{22} & 0 \\
* & * & -I
\end{array}\right]<0,
$$

where

$$
\begin{aligned}
& \Sigma_{11}=A^{T} \widetilde{P}_{1}+\widetilde{P}_{1} A+\widetilde{Q}_{1}+3 a^{2} I-\alpha \widetilde{P}_{1}, \\
& \Sigma_{12}=\widetilde{P}_{1} B, \quad \Sigma_{13}=\widetilde{P}_{1}, \\
& \Sigma_{22}=-(1-\mu) e^{\alpha h_{1}} \widetilde{Q}_{1}+3 b^{2} I .
\end{aligned}
$$

Then, along the trajectory of system (3.1),

$$
V(t) \leq e^{\alpha t} V(0)+3 e^{\alpha t} \int_{0}^{t} \beta^{2}(s) e^{-\alpha s} d s .
$$

Proof. Taking the derivative of $V(t)$ along the trajectory of system (3.1), it follows from (2.2), (2.3), and Lemma 3.1 that

$$
\begin{aligned}
\dot{V}_{1}(t)= & 2 x^{T}(t) \widetilde{P}_{1} \dot{x}(t) \\
= & 2 x^{T}(t) \widetilde{P}_{1}[A x(t)+B x(t-h(t))+f(t, x(t), x(t-h(t)))] \\
= & 2 x^{T}(t) \widetilde{P}_{1} A x(t)+2 x^{T}(t) \widetilde{P}_{1} B x(t-h(t))+2 x^{T}(t) \widetilde{P}_{1} f(t, x(t), x(t-h(t))) \\
\leq & x^{T}(t)\left(A^{T} \widetilde{P}_{1}+\widetilde{P}_{1} A\right) x(t)+2 x^{T}(t) \widetilde{P}_{1} B x(t-h(t)) \\
& +f^{T}(t, x(t), x(t-h(t))) f(t, x(t), x(t-h(t)))+x^{T}(t) \widetilde{P}_{1}^{2} x(t) \\
\leq & x^{T}(t)\left(A^{T} \widetilde{P}_{1}+\widetilde{P}_{1} A+\widetilde{P}_{1}^{2}+3 a^{2} I\right) x(t)+2 x^{T}(t-h(t)) B^{T} \widetilde{P}_{1} x(t) \\
& +3 b^{2} x^{T}(t-h(t)) x(t-h(t))+3 \beta^{2}(t)
\end{aligned}
$$


and

$$
\begin{aligned}
\dot{V}_{2}(t)= & \alpha V_{2}(t)+e^{\alpha t}\left[x^{T}(t) e^{-\alpha t} \widetilde{Q}_{1} x(t)\right. \\
& \left.-x^{T}(t-h(t)) e^{-\alpha(t-h(t))} \widetilde{Q}_{1} x(t-h(t))(1-\dot{h}(t))\right] \\
\leq & \alpha V_{2}(t)+x^{T}(t) \widetilde{Q}_{1} x(t)-(1-\mu) x^{T}(t-h(t)) e^{\alpha h_{1}} \widetilde{Q}_{1} x(t-h(t)) .
\end{aligned}
$$

Combining (3.4) and (3.5), we conclude that

$$
\begin{aligned}
\dot{V}(t)-\alpha V(t) \leq & {\left[\begin{array}{c}
x(t) \\
x(t-h(t))
\end{array}\right]^{T}\left[\begin{array}{cc}
\Sigma_{11}+\widetilde{P}_{1}^{2} & \Sigma_{12} \\
* & \Sigma_{22}
\end{array}\right]\left[\begin{array}{c}
x(t) \\
x(t-h(t))
\end{array}\right] } \\
& +3 \beta^{2}(t) \\
\leq & 3 \beta^{2}(t)
\end{aligned}
$$

when using (3.2) and the Schur complement formula. Therefore, we arrive at (3.3). This completes the proof.

The following theorem presents FTS conditions for switched delay system (2.1).

Theorem 3.3. For any $i \in \Lambda$, let $\widetilde{P}_{1, i}=R^{1 / 2} P_{1, i} R^{1 / 2}, \widetilde{Q}_{1, i}=R^{1 / 2} Q_{1, i} R^{1 / 2}$. Suppose that there exist matrices $P_{1, i}>0, Q_{1, i}>0$, and $\alpha>0$ such that

$$
\Phi=\left[\begin{array}{ccc}
\phi_{11} & \phi_{12} & \phi_{13} \\
* & \phi_{22} & 0 \\
* & * & -I
\end{array}\right]<0,
$$

where

$$
\begin{aligned}
& \phi_{11}=A_{i}^{T} \widetilde{P}_{1, i}+\widetilde{P}_{1, i} A_{i}+\widetilde{Q}_{1, i}+3 a^{2} I-\alpha \widetilde{P}_{1, i}, \\
& \phi_{12}=\widetilde{P}_{1, i} B_{i}, \quad \phi_{13}=\widetilde{P}_{1, i}, \\
& \phi_{22}=-(1-\mu) e^{\alpha h_{1}} \widetilde{Q}_{1, i}+3 b^{2} I,
\end{aligned}
$$

and

$$
\left(\lambda_{2}+h_{2} e^{\alpha h_{2}} \lambda_{3}\right) c_{1}+3 \int_{0}^{T} \beta^{2}(s) e^{-\alpha s} d s<c_{2} e^{-\alpha T} \lambda_{1}
$$

If the ADT of the switching signal $\sigma(t)$ satisfies

$$
\tau_{a}>\tau_{a}^{*}=\frac{T \ln \rho}{\ln \left(\lambda_{1} c_{2}\right)-\ln \left[\left(\lambda_{2}+h_{2} e^{\alpha h_{2}} \lambda_{3}\right) c_{1}\right]-\alpha T},
$$

then system (2.1) is FTS with respect to $\left(c_{1}, c_{2}, T, \sigma(t), R\right)$, where $\rho \geq 1, P_{1, i} \leq \rho P_{1, j}$, $Q_{1, i} \leq \rho Q_{1, j}, \forall i, j \in \Lambda, \lambda_{1}=\min _{i \in \Lambda}\left\{\lambda_{\min }\left(P_{1, i}\right)\right\}, \lambda_{2}=\max _{i \in \Lambda}\left\{\lambda_{\max }\left(P_{1, i}\right)\right\}$, and $\lambda_{3}=$ $\max _{i \in \Lambda}\left\{\lambda_{\max }\left(Q_{1, i}\right)\right\}$.

Proof. Define a Lyapunov-Krasovskii function by

$$
V(t)=V_{\sigma(t)}(t)=V_{1, \sigma(t)}(t)+V_{2, \sigma(t)}(t),
$$

where

$$
V_{1, \sigma(t)}(t)=x^{T}(t) \widetilde{P}_{1, \sigma(t)} x(t), \quad V_{2, \sigma(t)}(t)=\int_{t-h(t)}^{t} x^{T}(s) e^{\alpha(t-s)} \widetilde{Q}_{1, \sigma(s)} x(s) d s,
$$

and $\widetilde{P}_{1, i}$ and $\widetilde{Q}_{1, i}$ are positive definite matrices to be determined.

Step 1. Let $t \in\left[t_{k}, t_{k+1}\right)$. By virtue of (3.7) and Lemma 3.2, we have

$$
V(t)=V_{\sigma(t)}(t)<e^{\alpha\left(t-t_{k}\right)} V_{\sigma\left(t_{k}\right)}\left(t_{k}\right)+3 \int_{t_{k}}^{t} \beta^{2}(s) e^{\alpha(t-s)} d s .
$$

Since $\rho \geq 1, P_{1, i} \leq \rho P_{1, j}, Q_{1, i} \leq \rho Q_{1, j}, \forall i, j \in \Lambda$, and $\widetilde{P}_{1, i}=R^{1 / 2} P_{1, i} R^{1 / 2}, \widetilde{Q}_{1, i}=$ $R^{1 / 2} Q_{1, i} R^{1 / 2}$, we obtain

$$
\widetilde{P}_{1, i} \leq \rho \widetilde{P}_{1, j}, \quad \widetilde{Q}_{1, i} \leq \rho \widetilde{Q}_{1, j} .
$$


Without loss of generality, assume that $\sigma\left(t_{k}\right)=i, \sigma\left(t_{k}^{-}\right)=j$ at switching instant $t_{k}$, where $\sigma\left(t_{k}^{-}\right)=\lim _{\Delta t \rightarrow 0^{-}} \sigma\left(t_{k}+\Delta t\right)$. Noticing that $x\left(t_{k}\right)=x\left(t_{k}^{-}\right)$, we get

$$
V_{\sigma\left(t_{k}\right)}\left(t_{k}\right) \leq \rho V_{\sigma\left(t_{k}^{-}\right)}\left(t_{k}^{-}\right),
$$

where $x\left(t_{k}^{-}\right)=\lim _{\Delta t \rightarrow 0^{-}} x\left(t_{k}+\Delta t\right)$. It follows from (3.10) and (3.11) that

$$
V(t)<e^{\alpha\left(t-t_{k}\right)} \rho V_{\sigma\left(t_{k}^{-}\right)}\left(t_{k}^{-}\right) .
$$

Step 2. For any $t \in(0, T)$, letting $N$ be the switching number of $\sigma(t)$ over $(0, T)$, then $N_{\sigma}(0, t) \leq N$. Using the iterative method in Step 1, we conclude that

$$
\begin{aligned}
V(t) \leq & e^{\alpha\left(t-t_{k}\right)} \rho V_{\sigma\left(t_{k}^{-}\right)}\left(t_{k}^{-}\right)+3 e^{\alpha t} \int_{t_{k}}^{t} \beta^{2}(s) e^{-\alpha s} d s \\
\leq & \cdots \leq e^{\alpha t} \rho^{k} V_{\sigma(0)}(0)+3 e^{\alpha t} \rho^{k} \int_{0}^{t_{1}} \beta^{2}(s) e^{-\alpha s} d s \\
& +\cdots+3 e^{\alpha t} \int_{t_{k}}^{t} \beta^{2}(s) e^{-\alpha s} d s \\
= & e^{\alpha t} \rho^{k} V_{\sigma(0)}(0)+3 e^{\alpha t} \rho^{k} \int_{0}^{t} \beta^{2}(s) e^{-\alpha s} d s \\
\leq & e^{\alpha t} \rho^{k}\left(V_{\sigma(0)}(0)+3 \int_{0}^{T} \beta^{2}(s) e^{-\alpha s} d s\right) .
\end{aligned}
$$

Note that $k \leq N \leq T / \tau_{a}$. Then

$$
V(t) \leq e^{\alpha t} \rho^{T / \tau_{a}}\left(V_{\sigma(0)}(0)+3 \int_{0}^{T} \beta^{2}(s) e^{-\alpha s} d s\right) .
$$

On the other hand,

$$
\begin{aligned}
V(t) \geq & x^{T}(t) \widetilde{P}_{1, \sigma(t)} x(t) \geq \lambda_{\min }\left(P_{1, \sigma(t)}\right) x^{T}(t) R x(t) \geq \lambda_{1} x^{T}(t) R x(t), \\
V_{\sigma(0)}(0)= & x^{T}(0) \widetilde{P}_{1, \sigma(0)} x(0)+\int_{-h(0)}^{0} x^{T}(s) e^{-\alpha s} \widetilde{Q}_{1, \sigma(0)} x(s) d s \\
\leq & \lambda_{\max }\left(P_{1, \sigma(0)}\right) x^{T}(0) R x(0) \\
& +h_{2} e^{\alpha h_{2}} \lambda_{\max }\left(Q_{1, \sigma(0)}\right) \sup _{-h(0) \leq s \leq 0}\left\{x^{T}(s) R x(s)\right\} \\
\leq & \left(\lambda_{\max }\left(P_{1, \sigma(0)}\right)+h_{2} e^{\alpha h_{2}} \lambda_{\max }\left(Q_{1, \sigma(0)}\right)\right) \sup _{-h_{2} \leq s \leq 0}\left\{x^{T}(s) R x(s)\right\} \\
\leq & \left(\lambda_{2}+h_{2} e^{\alpha h_{2}} \lambda_{3}\right) c_{1} .
\end{aligned}
$$

Combining (3.12), (3.13), and (3.14), we arrive at

$$
\begin{aligned}
x^{T}(t) R x(t) & \leq \frac{V(t)}{\lambda_{1}} \leq \frac{e^{\alpha T} \rho^{\frac{T}{\tau_{a}}}\left(V_{\sigma(0)}(0)+3 \int_{0}^{T} \beta^{2}(s) e^{-\alpha s} d s\right)}{\lambda_{1}} \\
& \leq \frac{e^{\alpha T} \rho^{\frac{T}{\tau_{a}}}\left[\left(\lambda_{2}+h_{2} e^{\alpha h_{2}} \lambda_{3}\right) c_{1}+3 \int_{0}^{T} \beta^{2}(s) e^{-\alpha s} d s\right]}{\lambda_{1}} .
\end{aligned}
$$

The following proof can be divided into two cases.

Case 1. Assume that $\rho=1$. It follows from (3.8) and (3.15) that

$$
x^{T}(t) R x(t)<c_{2} e^{-\alpha T} e^{\alpha T}=c_{2} .
$$

Case 2. Suppose that $\rho>1$. Using (3.8), we obtain

$$
\ln \left(\lambda_{1} c_{2}\right)-\ln \left[\left(\lambda_{2}+h_{2} e^{\alpha h_{2}} \lambda_{3}\right) c_{1}+3 \int_{0}^{T} \beta^{2}(s) e^{-\alpha s} d s\right]-\alpha T>0 .
$$


By virtue of (3.9), we get

$$
\begin{aligned}
\frac{T}{\tau_{a}} & <\frac{\ln \left(\lambda_{1} c_{2}\right)-\ln \left[\left(\lambda_{2}+h_{2} e^{\alpha h_{2}} \lambda_{3}\right) c_{1}+3 \int_{0}^{T} \beta^{2}(s) e^{-\alpha s} d s\right]-\alpha T}{\ln \rho} \\
& =\frac{\ln \frac{c_{2} \lambda_{1} e^{-\alpha T}}{\left(\lambda_{2}+h_{2} e^{\left.\alpha h_{2} \lambda_{3}\right) c_{1}+3 \int_{0}^{T} \beta^{2}(s) e^{-\alpha s} d s}\right.}}{\ln \rho} .
\end{aligned}
$$

Substituting (3.17) into (3.15), we deduce that

$$
\begin{aligned}
x^{T}(t) R x(t)< & \frac{\left(\lambda_{2}+h_{2} e^{\alpha h_{2}} \lambda_{3}\right) c_{1}+3 \int_{0}^{T} \beta^{2}(s) e^{-\alpha s} d s}{\lambda_{1}} e^{\alpha T} \\
& \times \frac{c_{2} \lambda_{1} e^{-\alpha T}}{\left(\lambda_{2}+h_{2} e^{\alpha h_{2}} \lambda_{3}\right) c_{1}+3 \int_{0}^{T} \beta^{2}(s) e^{-\alpha s} d s} \\
= & c_{2} .
\end{aligned}
$$

Taking into account that the trajectory of system (2.1) remains continuous at instant $T$, we conclude that (3.16) and (3.18) hold for all $t \in[0, T]$. The proof is complete.

\section{Numerical example}

The following example illustrates applications of theoretical results presented in this paper.

Example 4.1. Consider a switched system

$$
\dot{x}(t)=A_{\sigma(t)} x(t)+B_{\sigma(t)} x(t-h(t))+f_{\sigma(t)}(t, x(t), x(t-h(t)))
$$

with

$$
\begin{array}{ccc}
h(t)=0.9 \sin t+0.1, \quad x(t)=\left[\begin{array}{c}
2 \\
-1 \\
-2
\end{array}\right], \quad t \in[-0.1,0], & \\
A_{1}=\left[\begin{array}{ccc}
-2.7 & 1.7 & 0 \\
1.3 & -1 & 0.7 \\
0.7 & 1 & -0.6
\end{array}\right], & A_{2}=\left[\begin{array}{ccc}
1 & -1 & 0 \\
0.7 & 0 & -0.6 \\
1.7 & 0 & -1.7
\end{array}\right], \\
B_{1}=\left[\begin{array}{ccc}
1.5 & -1.7 & 0.1 \\
-1.3 & 1 & -0.3 \\
-0.7 & 1 & 0.6
\end{array}\right], & B_{2}=\left[\begin{array}{ccc}
-0.5 & 0 & 0.1 \\
1.3 & -1 & -0.6 \\
1.5 & 0.1 & 1.8
\end{array}\right],
\end{array}
$$

and

$$
f_{1}=\left[\begin{array}{c}
e^{-t} \\
4 \\
-3
\end{array}\right], \quad f_{2}=\left[\begin{array}{c}
-\left(x_{2}(t)\right)^{\frac{2}{3}}\left(x_{1}(t-h(t))\right)^{\frac{1}{3}} \\
e^{-t} \\
\frac{x_{1}(t)+x_{3}(t-h(t))}{1+\left(x_{1}(t)\right)^{2}}
\end{array}\right] .
$$

The values of $c_{1}, c_{2}, T$, and matrix $R$ are given as follows:

$$
c_{1}=9, \quad c_{2}=49, \quad T=13, \quad R=I .
$$

By virtue of Theorem 3.3, system (4.1) is FTS for any switched signal which satisfies $\tau_{a}>\tau_{a}^{*}$, with respect to $(9,49,13, \sigma(t), I)$.

Remark 4.2. It follows from Figure 1 that system (4.1) is FTS, and it is more effective than that of [10].

Remark 4.3. In this paper, using the ADT approach and constructing Lyapunov-Krasovskii functions, we have established new FTS criteria for a class of switched delay systems with nonlinear perturbations (2.1). The conditions obtained are easy to verify, and the technique proposed essentially simplify the process of investigation of switched delay systems. 


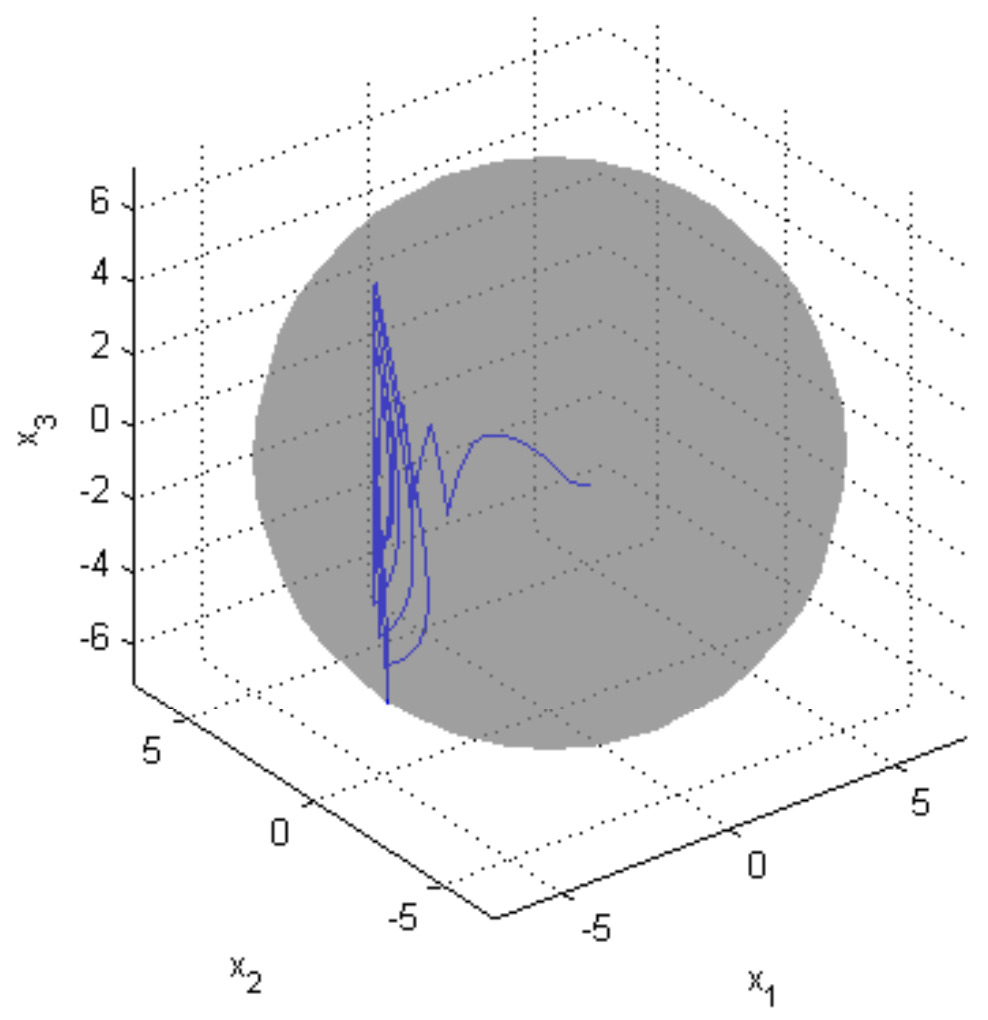

Figure 1. The state trajectory of system (4.1) under switching signals.

It would be of interest to study uniformly FTS of $(2.1)$. Similarly to $[3,7,17]$, it would also be interesting to investigate convergence and oscillatory properties of switched systems.

\section{Acknowledgements}

The authors are grateful to the editors and anonymous referees for a very thorough reading of the manuscript and for kindly prompting improvements in presentation. This research is supported by NNSF of P. R. China (Grant Nos. 61633015, 61273097, 61527809, and 61503171), CPSF (Grant No. 2015M582091), NSF of Shandong Province (Grant No. ZR2016JL021), KRDP of Shandong Province (Grant No. 2017CXGC0701), DSRF of Linyi University (Grant No. LYDX2015BS001), and the AMEP of Linyi University, P. R. China.

\section{References}

[1] F. Amato, R. Ambrosino, M. Ariola, and C. Cosentino, Finite-time stability of linear time-varying systems with jumps, Automatica J. IFAC 45, 1354-1358, 2009.

[2] F. Amato, M. Ariola, and P. Dorato, Finite-time control of linear systems subject to parametric uncertainties and disturbances, Automatica J. IFAC 37, 1459-1463, 2001.

[3] M. Bohner, T.S. Hassan, and T. Li, Fite-Hille-Wintner-type oscillation criteria for second-order half-linear dynamic equations with deviating arguments, Indag. Math. (N.S.) 29, 548-560, 2018.

[4] P. Dorato, Short-time stability in linear time-varying systems, in: Proceedings of the IRE International Convention Record Part 4, New York, pp. 83-87, 1961.

[5] J.P. Hespanha and A.S. Morse, Stability of switched systems with average dwell-time, in: Proceedings of the 38th IEEE Conference on Decision and Control, Phoenix, pp. 2655-2660, 1999. 
[6] Z. Ji, L. Wang, and X. Guo, On controllability of switched linear systems, IEEE Trans. Automat. Control 53, 796-801, 2008.

[7] T. Li and Yu.V. Rogovchenko, Oscillation criteria for second-order superlinear Emden-Fowler neutral differential equations, Monatsh. Math. 184, 489-500, 2017.

[8] D. Liberzon, Switching in Systems and Control (Birkhäuser, Boston, 2003).

[9] H. Lin and P.J. Antsaklis, Stability and stabilizability of switched linear systems: a survey on recent results, IEEE Trans. Automat. Control 54, 308-322, 2009.

[10] X. Lin, H. Du, and S. Li, Finite-time boundedness and $L_{2}$-gain analysis for switched delay systems with norm-bounded disturbance, Appl. Math. Comput. 217, 5982-5993, 2011.

[11] X. Lin, X. Li, S. Li, and Y. Zou, Finite-time boundedness for switched systems with sector bounded nonlinearity and constant time delay, Appl. Math. Comput. 274, 2540, 2016.

[12] X. Liu, Stability analysis of a class of nonlinear positive switched systems with delays, Nonlinear Anal. Hybrid Syst. 16, 1-12, 2015.

[13] J. Liu, J. Lian, and Y. Zhuang, Output feedback $L_{1}$ finite-time control of switched positive delayed systems with MDADT, Nonlinear Anal. Hybrid Syst. 15, 11-22, 2015.

[14] D. Liu, X. Liu, and G. Xiao, Stability and $L_{2}$-gain analysis for switched systems with interval time-varying delay, IET Control Theory Appl. 9, 1644-1652, 2015.

[15] F. Pan, X.-B. Chen, and L. Lin, Practical stability analysis of stochastic swarms, in: The 3rd International Conference on Innovative Computing, Information and Control, Dalian, pp. 32-36, 2008.

[16] Y. Tian, Y. Cai, Y. Sun, and H. Gao, Finite-time stability for impulsive switched delay systems with nonlinear disturbances, J. Franklin Inst. 353, 3578-3594, 2016.

[17] P. Wang and X. Liu, Rapid convergence for telegraph systems with periodic boundary conditions, J. Funct. Spaces 2017, 1-10, 2017.

[18] M. Xiang and Z. Xiang, Exponential stability of discrete-time switched linear positive systems with time-delay, Appl. Math. Comput. 230, 193-199, 2014.

[19] Y. Zhang, M. Wang, H. Xu, and K.L. Teo, Global stabilization of switched control systems with time delay, Nonlinear Anal. Hybrid Syst. 14, 86-98, 2014.

[20] K. Zhou and P.P. Khargonekar, Robust stabilization of linear systems with normbounded time-varying uncertainty, Systems Control Lett. 10, 17-20, 1988.

[21] G. Zong, R. Wang, W.X. Zheng, and L. Hou, Finite-time stabilization for a class of switched time-delay systems under asynchronous switching, Appl. Math. Comput. 219, 5757-5771, 2013. 